\title{
Leveraging Trust Relations to Improve Academic Patent Recommendation
}

\author{
Yuwen Chen \\ School of Management, \\ University of Science and \\ Technology of China \\ chenyw16@mail.ustc.edu.cn \\ Xiaoming Huang \\ Department of Information Systems, \\ City University of Hong Kong \\ xmhuang6-c@my.cityu.edu.hk
}

\author{
Jian Ma \\ Department of Information Systems, \\ City University of Hong Kong \\ isjian@cityu.edu.hk
}

Jin Qin

School of Management,

University of Science and

Technology of China

qjin@ustc.edu.cn

\author{
Peihu Zhu \\ Department of Information Systems, \\ City University of Hong Kong \\ peihuzhu2@cityu.edu.hk
}

\begin{abstract}
Academic patent trading is one of the important ways for university technology transfer. Compared to industry patent trading, academic patent trading suffers from a more serious information asymmetric problem. It needs a recommendation service to help companies identify academic patents that they want to pay. However, existing recommendation approaches have limitations in facilitating academic patent trading in online patent platforms because most of them only consider patent-level characteristics. A high trust degree of a company towards academic patents can alleviate the information asymmetry and encourage trading. This study proposes a novel academic patent recommendation approach with a hybrid strategy, combining citation-based relevance, connectivity, and trustworthiness. An offline experiment is conducted to evaluate the performance of the proposed recommendation approach. The results show that the proposed method performs better than the baseline methods in both accuracy and ranking.
\end{abstract}

\section{Introduction}

Patent trading is a popular and important way to transfer technology and commercialize innovation among companies and institutions [1,2]. Companies can obtain using rights of patents and hold a competitive market position through patent trade [2]. Most universities have their own University Office of Technology Transfer offices (UOTT) to support technology transfer activities. However, the capacity of UOTTs is limited in patent marketing due to the

\footnotetext{
${ }^{1}$ http://www.yet2.com

${ }^{2}$ http://www.tynax.com
}

shortages of staff and budget [3], which makes the commercialization of academic patents a challenging task. In recent years, online patent platforms such as Yet $2^{1}$, Tynax ${ }^{2}$, and InnoCity ${ }^{3}$ are emerging as a new form of patent intermediary [4-6]. One of the goals of these platforms is to promote offline trading by creating an effective way to connect patent owners and companies. Company users in the platform can seek patents that they need without the limitations of time and place. Through the online platform, inventors or patent owners can upload their patents. It enables universities and their researchers to monetize their patented knowledge assets with less marketing cost.

However, compared to non-university patents driven by the established market, it is more challenging for companies to identify academic patents in the online environment. A reason is that most university inventions are driven by high-tech innovation and their potential market space is often unknown [3]. Companies are more uncertain about university patents' potential market and business value than non-university patents, which becomes an obstacle to academic patent trading. The first reason is the information asymmetry problem from the inventor. There is an imbalance between the inventor and the company in their knowledge of a specific patent. Such imbalance even shows a trend of exacerbating when the patents are new [7-9]. Inventors often equip more knowledge than described in the patent specification, including not only technical properties but also intangible issues such as the difference between similar patents and the commercial value of the patent. On the other side, patent-seeking companies have little information related to the utility of the patent. The inventors can exaggerate the business value when they

\footnotetext{
${ }^{3}$ https://www.innocity.com/onlineweb/
} 
want to commercialize their patented inventions, although their patents may not be as valuable as they claim [10]. The second reason for the uncertainty is the technology transfer policy of the university [3]. Universities with rich experience in technology transfer tend to develop more encourage policies for the collaboration between researchers and companies, which is beneficial for companies to obtain more information about the academic patents and exploit their business value. Therefore, companies' uncertainty of academic patents easily causes trust issues of patent trading, which is one of the main obstacles to reaching a patent-transaction agreement.

In order to help the decision-maker to discover appropriate patents efficiently, several patent recommendation approaches were proposed for general patent finding [11-14], citation [15, 16], patent maintenance [17], and patent technology trade [6, 18]. When seeking patents through online trading platforms, companies often consider multiple dimensions before decision-making. These works mainly consider the features of patents but neglect the trust relations of companies with inventors and owners of patents.

In the technology market, a high level of trust relations between trading parties encourages more opportunities for successful patent trading and helps to reduce trading costs $[7,19]$. In a patent trading platform, the trust of companies towards a patent is transferred from intermediaries to someone who invents or owns the patent. Especially for academic patents, companies tend to trust the corresponding inventors and patent owners who have a high level of expertise or rich experience in patent transactions.

This study aims to develop an effective approach for academic patent trading recommendations under online platforms. In this study, the degree of the trust relationship is strengthened by historical interactions among transaction parties and the trustworthiness of the inventor and patent owner. Compared to the previous recommendation approach with only patent analysis, our approach focuses more on recommending academic patents to facilitate university technology transfer and additionally captures the characteristic information of the patent owner and inventor from the platform. A hybrid strategy is adopted for the recommendation with two main stages of candidates filtering and ranking. The candidate set is firstly generated according to the classification information and then ranked based on the citation network and trust information.

The rest of the paper is organized as follows. Section 2 reviews related work. Section 3 describes the proposed trust-enhanced recommendation approach for academic patent trading. In Section 4 , we conduct an offline experiment to evaluate the effectiveness of the proposed method, and the experiment results are presented. Section 5 concludes this study and summarizes contributions and limitations.

\section{Related work}

In recent years, application study is pointed out as the popular research focus in the recommendation system because it offers great opportunities and challenges in many domains such as business, government, and education [20]. Online platforms for patent trading have appeared as a new type of patent intermediary for several years [4]. The early research on patent recommendations mainly focused on the characteristics of the patent itself. With data accumulated in the online environment, it becomes a chance to offer better recommendations by utilizing the online information. Therefore, considering more characteristics of trust relations before generating the recommendation list is another way to improve the performance of a patent recommendation service. Our work is related to recommendation technologies, application of trust relations in recommendation approaches, and measurement of trust degree based on historical interaction records. Related literature is reviewed as follows.

\subsection{Patent recommendation}

Recommendation services in online platforms such as Amazon, Netflix, and similar others help increase transaction opportunities, by offering users a helping hand to find the items they are interested in [20]. Several patent recommendation methods have been proposed for various application backgrounds such as patent citation, patent maintenance, and general finding [6, 16$18,21]$. Current patent recommendation methods can be categorized as content-based, collaborative filteringbased, and hybrid methods.

Content-based methods recommend patents to a user by matching textual information of patents with the content of the user's query. The textual information of a patent can be classification code, title, abstract, and claims. Recent content-based methods are proposed to solve the keyword-mismatch problem [22] by generating IPC-based indexing vocabulary [23], extending the query construction [13], introducing semantic concept [24, 25], and constructing a heterogeneous topic model with word embedding [21]. Moreover, previous research shows that utilizing classification information and quality evaluation of the retrieved documents can also improve the performance of patent recommendations [17, 23].

Collaborative filtering is another mainstream type of recommendation algorithm, and it makes a recommendation based on the connectivity between 
users and items [20]. Collaborative filtering methods are developed mainly based on two assumptions. One assumption is that users with similar searching behaviors are interested in the same patent. Trappey et al. [11] developed a recommendation system that utilizes a user's operational history and adopts collaborative filtering algorithms based on users' behavior similarity. The other assumption is that users prefer patents similar to what they have already searched or purchased. Similar patents can be identified by random walking on different paths on patent citationbibliographic networks [16] and topics matching based on co-citation relations [26].

Researchers also explore hybrid recommendation methods that combine the advantage of both contentbased and collaborative filtering techniques for better recommendation performance. Considering that a patent document consists of textual description, citations, and bibliographic information, Oh et al. proposed a twostage framework for patent citation recommendation [15]. In the first stage, candidate patents are obtained by a content-based retrieval technique. In the second stage, the candidate list is ranked by a collaborative filtering model. In order to integrate various patent information, a patent trading recommendation mechanism based on heterogeneous information networks (HIN) has also been developed [6, 18]. The recommendation mechanism proposed by Wang et al. firstly constructs HIN according to various patent information and secondly recommends patents based on a meta-path similarity measurement [6]. The work of He et al. [18] additionally incorporated the patent's affiliation and its location into the HIN, and the recommendation model was trained based on weighted meta structures.

Previous patent recommendation approaches mainly focus on the content-based relevance and connectivity between companies and patents. Some components of these approaches can also be adopted to recommend academic patents. However, in the application of facilitating patent transactions through online marketplaces, these approaches have limitations in recommending patents that companies are willing to pay. On the one hand, when making a purchasing decision, companies consider both patent-level characteristics and human-level characteristics such as the owner's standing [27]. On the other hand, companies also face high search costs and adverse selection risks because the ratio of online low-quality and high-quality patents on online marketplaces is often very high [4]. Therefore, our study additionally considers the characteristics of the patent owner and inventor from the perspective of trust relations in academic patent trading.

\subsection{Trust relations in academic patent trading}

Trust relationships have been used to improve the performance of recommendations, for it is more likely to influence one's decision purchase than website advertising in the real world [20].

Prior works also suggested that using trust information can strengthen the ability to make accurate recommendations, especially when solving cold start and data sparsity problems [28]. In the current literature, trust-based filtering methods for recommendation mainly employ explicit trust and implicit trust for trust computation [29]. In explicit trust filtering methods, the trust degree of a user to others is explicitly indicated by the user $[30,31]$. They collect trust values directly indicated by users to construct trust networks or paths. However, the acquisition of explicit trust requires additional labor of users to provide trust score to others, and the filtering method is not effective until a new user build up their web of trust [28, 29]. The acquisition of the explicit score limits the applicability of explicit trust filtering methods. In recent years, the implicit trust filtering methods are more popular because it is more feasible to use with less manual cost [32]. Most negotiations for patent transactions are under conditions of secrecy, and the trading details are rarely disclosed after the transaction is done [33]. Therefore, it is hard and almost impossible to ask the parties involved in the patent transaction to give a trust score for each other for others' references, which is different from general ecommerce environments. In this paper, implicit trust is taken into account for the patent recommendation. The implicit trust filtering method obtains the trust value inferred from other available trust-sensitive information such as the user similarity on item rating [34, 35], the proportion of making successful recommendations [36], and the ability to deliver a reliable recommendation in the past [28, 29].

In the issue of university-industry (UI) technology transfer, extant research has explored the determinations of university technology commercialization [37], performance measurement of UI alliance [38, 39], and trust-building in UI collaborations [40]. Successful UI technology transfer activities mostly depend on trust cultivation to bridge the knowledge divide between academe and industry. Both tie strength and partner reputation help build trust in UI collaborations [40]. Tie strength measures the frequency of historical collaborations between partners, and partner reputation is determined by the university partner's research quality and transfer performance in the past. Besides, a company's trust in a potential counterparty can be gained from their historical interactions $[7,19]$ and the expertise of the counterparty [41]. 
In the context of academic patent trading, university partners could be researchers as inventors and universities as patent owners. Researchers' characteristics, including scientific impact, project experience, and patent trading experience, play an important role in successful technology transfer from university to industry [42]. Scientific productivity and quality of university in terms of technology transfer also encourage the university technology commercialization activities [37]. Multiple indicators that include industry income and the number of transfer contracts are suggested to measure the UI knowledge transfer performance [39].

Previous methods concerning patent recommendation rarely consider trust information. During the selection process for academic patents in an online environment, the trust of a company in an academic patent especially plays an important role in a company's decision of whether to pay for the patent. As a technology transfer way from university to industry, academic patent trading also depends on trust cultivation to ease the information asymmetry problem. Motivated by facilitating patent transactions, this study transfers the trust of companies towards a patent to someone who has invented or hold the patent and develops a hybrid recommendation approach combining relevance analysis and trust analysis.

\section{The hybrid trust-enhanced recommendation approach}

\subsection{Overview of the recommendation approach}

In an online patent platform for patent trading, there are a set of academic patents $P=\left\{p_{1}, p_{2}, \ldots, p_{N}\right\}$ and a set of company users $C=\left\{c_{1}, c_{2}, \ldots, c_{M}\right\}$ who want to buy academic patents. The proposed recommendation approach can help user $c_{i} \in C$ with patent selection efficiently by taking relevance and trust information into account. Figure 1 shows the overview of the proposed hybrid approach for the academic patent trading recommendation. The approach mainly contains two stages. In the filtering stage, a candidate set of patents is generated for a target company through a classificationbased filtering method. In the ranking stage, we calculate citation-based connectivity, interactional connectivity, and trustworthiness of each candidate patent for the company. Then, we integrate the measurement scores of the three dimensions by a trust analysis model and Logistic Regression model (LR) to obtain the ranking scores to rank patents in the candidate list. Top-K patents are selected to form the final recommendation list.

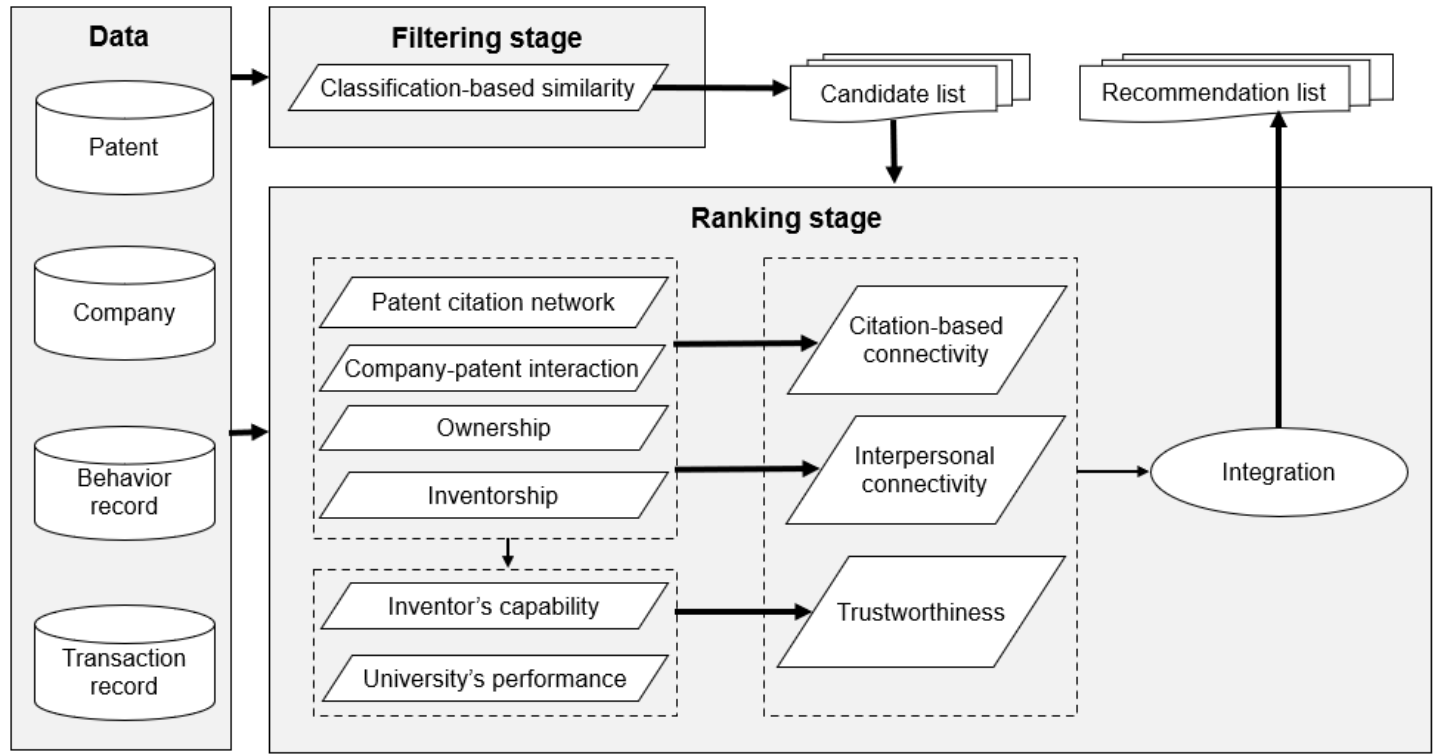

Figure 1. Overview of the recommendation approach

\subsection{Filtering stage}

The candidate patents are extracted according to the classification matching between the target company and academic patents uploaded on the platform. It improves the efficiency of the recommendation by initially removing irrelevant patents in terms of the technology field.
The classification code of a patent, such as the International Patent Classification (IPC) code and Cooperative Patent Classification (CPC) code, reveals to which technology area the patent belongs. A company's historical patents and online behaviors construct the latent interests of the company. Thus, we define the classification-based profile of company $c$ as a set of classification codes of all patents it owns, uses, 
and consults in the past five years, which is represented as a set of classification codes $c C I D=$ $\left\{c C I D_{1}, c C I D_{2}, \ldots, c C I D_{n_{c}}\right\}$. For one patent can also cover several classifications, we denote the classification-based profile of patent $p$ as $p C I D=$ $\left\{p C I D_{1}, p C I D_{2}, \ldots, p C I D_{n_{p}}\right\}$. Patent $p$ can become an initial candidate when it meets the technology fields of patent seeker $c$, i.e.,

$$
p C I D \subset c C I D \text {. }
$$

\subsection{Ranking stage}

We rank the candidate patents in the ranking stage based on three dimensions, citation-based connectivity, interpersonal connectivity, and trustworthiness. The first two dimensions are calculated using the Personalized PageRank (PPR) model, and the last one by trust analysis. Then, a Logistic Regression model is adopted to integrate the above measurement results. Candidate patents are recommended according to their aggregated scores.

\subsubsection{Citation-based and interpersonal} connectivity. In the patent trading platform, company users can build connections with academic patents through trading activities and online consulting behaviors. Ownership, inventorship, and cited patents can be found in the meta-information of patent documents. We collect the above information to calculate the connectivity between companies and patents, as shown in Figure 2.

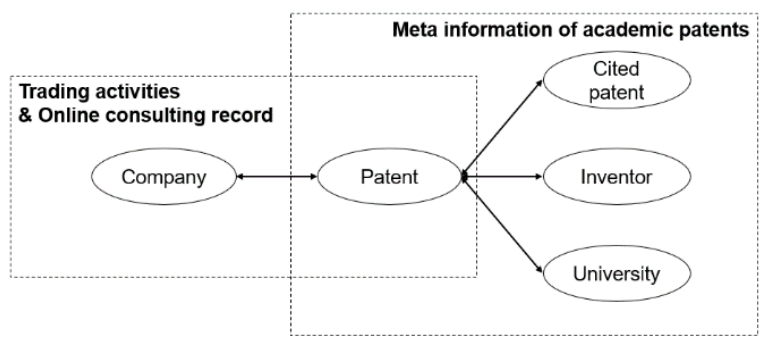

\section{Figure 2. Information for the connectivity} calculation

Citation linkages between patents reveal their technological relevance [16]. Besides, the interpersonal connectivity of the company towards inventors and patent owners is also helpful in making a successful transaction of academic patents [3]. Thus, we calculate the citation-based and interpersonal connectivity between companies and patents. Firstly, we construct three kinds of tripartite networks $G_{c i t}=$ $\left\langle c_{0}, P, Q, E_{\text {hist }}, E_{\text {cit }}\right\rangle$ $\left\langle c_{0}, P, R, E_{\text {hist }}, E_{\text {invent }}\right\rangle \quad, \quad$ and $\quad G_{\text {own }}=$ $\left\langle c_{0}, P, U, E_{\text {hist }}, E_{\text {own }}\right\rangle$ for a target company $c_{0}$. Patent set $P$ contains candidate patents and patents that have previous interactions with company $c_{0}$. Sets $Q, R$, and $U$ respectively represent the corresponding citing patents, inventors and patent owners. Edge set $E_{\text {hist }}$ represents historical interactions between company $c_{0}$ and patents. $E_{\text {cite }}, E_{\text {invent }}$, and $E_{\text {own }}$ are the edge sets representing relations of citation, invention, and owning, respectively. Secondly, we adopt a Personalized PageRank (PPR) model to calculate the connectivity degree between all candidate patents and the target company based on different paths. In each constructed tripartite network, the access possibility $P_{j}$ of node $j$ for each walk is calculated by,

$$
P_{j}=(1-\alpha) I_{j}+\alpha \sum_{i \in i n(j)} \frac{P_{i}}{|\operatorname{out}(i)|} .
$$

$I_{j}$ indicates whether the random walk will jump to the core code after a restart. $I_{j}=1$ if node $j$ is the core node, and otherwise $I_{j}=0$. Instead of $1 / N$ in the classical PageRank model, the introduction of $I_{j}$ ensures that the calculated result reflects the importance of each node in the network to the core node. in $(i)$ represents the set of all nodes that point to $i$, and $\operatorname{out}(i)$ represents the set of nodes to which $i$ points. Parameter $\alpha$ is the probability of continue walking to the next node. In the beginning, the access possibility of core node $c_{0}$ equals one, while other nodes are zero. After multiple walks, the stable access possibility of each node is obtained, which reflects the connectivity degree of each node to the core node. After random walks on three tripartite networks, we obtain varying connection degrees between candidate patents and company $c_{0}$ based on citation, inventor relation, and ownership relation. They are respectively represented by $R_{p c}^{\text {citation }}, R_{p c}^{\text {invention }}$, and $R_{p c}^{\text {ownership }}$.

3.2.2. Trustworthiness analysis. Trustworthiness assesses whether a trustee provides reliable information to trustors in a trust relation [35]. For an academic patent transaction, the trustworthiness of the corresponding inventor and university also encourages patent trading with companies. The trustworthiness of inventors is closely related to their capability of achieving a patent transaction, including the capability of trading, invention, research, and collaboration with companies $[3,17]$. The trading capability of inventor $r$ is measured according to his/her patent conversion rate $Q_{r}^{\text {trade }}=\left|T S_{r}\right| /\left|A S_{r}\right|$. If most of the patents invented by an inventor have transferred to others, it shows that the inventor is actively engaged in patent transfer activities. The invention capability $Q_{r}^{\text {invent }}$ of $r$ is assessed by the average citation frequency of patents invented by $r$. Citation frequency $Q_{p}=$ $e^{-\alpha\left(T_{y}-T_{p}\right)} C_{p}$ reflects patent $p$ 's influence, where $C_{p}$ is the number of times patent $p$ was cited from its 
publishing year $T_{p}$ to the current year $T_{y}$. The part $e^{-\alpha\left(T_{y}-T_{p}\right)}$ is the time decay function, where $\alpha>0$ is the attenuation constant. A researcher with highinfluence patents is more likely to be a trustworthy inventor. The research capability $Q_{r}^{\text {research }}$ of $r$ is assessed by using the corresponding $\mathrm{H}$-index because citation counts of research papers implicate the scientific impact [38]. The collaboration capability $Q_{r}^{\text {project }}$ of inventor $r$ is measured based on the number of projects in which $r$ has participated. After normalizing the above measurement results into the range of $[0,1]$, the trustworthiness of inventor $r$ is measured by,

$$
Q_{r}=\sum_{k \in K} \beta_{k} Q_{r}^{k} .
$$

$K=\{$ trade, invent, research, project $\}$ is the set of capability appellation. Parameters $\beta_{k}$ are used to assign the importance of the for kinds of capability, and $\sum_{k \in K} \beta_{k}=1$. In this study, we simply set $\beta_{k}=$ $1 / 4$ for each $k \in K$.

As owners of academic patents, universities often accumulate their trustworthiness in technology transfer activities by previous knowledge transfer performance [37, 39, 40]. In academic patent trading, the number of projects $Q_{u}^{\text {project }}$ and the number of patent transactions $Q_{u}^{\text {transfer }}$ are chosen as indicators of the trustworthiness of university $u$.

$$
Q_{u}=\frac{\lg \left(1+Q_{u}^{\text {project }}+Q_{u}^{\text {transfer }}\right)}{\max _{u \in U}\left(\lg \left(1+Q_{u}^{\text {project }}+Q_{u}^{\text {transfer }}\right)\right)} .
$$

3.2.3. Recommendation. The final ranking score is obtained by considering connectivity between patents and companies based on three kinds of relations and the trustworthiness of inventors and universities. Logistic regression (LR) analysis [43] can be adopted to assign weights to each relation. Let $\boldsymbol{X}_{\boldsymbol{p}}=$ $\left(R_{p c}^{\text {citation }}, R_{p c}^{\text {invention }}, R_{p c}^{\text {ownership }}, \max _{r \in R_{p}} Q_{r}^{p}, Q_{u}^{p}\right)$ be the input vector, where $R_{p}$ is the set of inventors of patent $p . Q_{r}^{p}$ and $Q_{u}^{p}$ represent the trustworthiness of the corresponding researcher $r$ and university $u$ of patent $p$. Then, the personalized ranking score $S R_{p c}$ for patent $p$ concerning company $c$ can be obtained by

$$
S R_{p c}=\frac{1}{1+e^{-\omega^{T} X_{p c}}} .
$$

$\boldsymbol{\omega}$ denotes the weight vector, which is obtained after LR training. Finally, candidate patents are ranked from highest to lowest score, and patents ranked in Top-K

\footnotetext{
${ }^{4}$ https://www.uspto.gov/learning-and-resources/electronic-dataproducts/patent-assignment-dataset
}

are extracted as the final recommendation list, where $\mathrm{K}$ is the number of patents in the list.

\section{Offline experiment}

\subsection{Experiment design}

We evaluate the recommendation performance of the proposed approach with Patent Assignment Dataset $^{4}$ and PatentsView Database ${ }^{5}$ from the United States Patent and Trademark Office (USPTO). Assignment records where patents were transferred from universities from the year 2012 to 2017 are firstly extracted. Companies with more than ten and less than five hundred assignments are chosen as target companies from these records. The assignment records of these companies are selected for the experiment. Totally 72 companies, 12290 academic patents, 8776 inventors, and 374 universities are included in our experiment.

We adopt three widely-used evaluation metrics precision, recall, and F1-score, to measure the accuracy of recommendation methods. Precision measures the number of patents that the company accepted in the recommendation list. Recall measures how many patents received by the company in the real world were predicted correctly. F1-score comprehensively considers Precision and Recall through weighted and average. They are described as follows,

$$
\begin{aligned}
& \operatorname{Pre} @ K=\frac{|A S \cap R S @ K|}{|R S @ K|}, \\
& R e c @ K=\frac{|A S \cap R S @ K|}{|A S|}, \\
& F 1 @ K=\frac{2 \times \text { Precision@K } \times \text { Recall@K }}{\text { Precision@K+Recall@K }},
\end{aligned}
$$

where $R S @ K$ is the set of patents in the top-K recommendation list, and $A S$ denotes the corresponding set of patents that happen trading activities with a company in the testing set. We also use Normalized Discounted Cumulative Gain (NDCG) to measure the ranking performance. It grades recommendation results on multiple levels of sequential coincidence, which is calculated by,

$$
N D C G @ K=\frac{1}{Z_{K}} \sum_{k=1}^{K} \frac{2^{S R_{k-1}}}{\log _{2}(k+1)} .
$$

$\sum_{k=1}^{K} \frac{2^{S R_{k-1}}}{\log _{2}(k+1)}$ is the Discounted Cumulative Gain (DCG) of top-K recommendation. $S R_{k}$ equals 1 when the company buys the $k$-th patent in the real world;

\footnotetext{
${ }^{5}$ http://www.patentsview.org/download/
} 
otherwise, it equals $0 . Z_{K}$ is the value of DCG under the perfect rank.

Three baseline methods are used for the comparison with our proposed trust-enhanced patent recommendation approach. The first baseline method is the classification-based method (represented as CB). It makes recommendations only based on the classification relevance of patents to the target company. The second one additionally utilized a patent citation network to find relevant patents after classification filtering (represented as CRS). The CRS scheme is initially proposed by [14], which utilizes citation links after content-based filtering to identify relevant patents further. In CRS, patents with high textual relevance become candidates, and they are ranked by using PPR model on the citation graph. The third baseline method is the patent recommendation without considering trustworthiness information (represented as PL). Similar to prior work of [6, 15], PL synthesizes various patent information, including contents, citations, and bibliographic information, to conduct recommendations, but it does not conduct the trustworthiness analysis (represented as PL). It generates candidates by content-based method and ranks the patent by PPR model on a citationbibliographic network. Compared to PL, our method (TE_PL) additionally considers researchers' and universities' trustworthiness for the academic patent recommendation.

\subsection{Experiment results}

This section analyzes the accuracy and ranking performance of different recommendation methods (i.e., CB, CRS, PL, and TE_PL). The experimental results of different recommendation sizes are presented in Figures 3-6, where $\mathrm{N}$ represents the recommendation size. The results show that both PL and TE_PL have noticeable improvements in accuracy and ranking compared with $\mathrm{CB}$ and $\mathrm{CRS}$. It proves that interpersonal connections mediated by patents, such as relations of invention and ownership in this study, play an essential role in affecting the recommendation performance in the context of patent trading. Moreover, our proposed method TE_PL ranks first on all four metrics. It reflects that the introduction of trustworthiness can also help improve the performance of academic patent trading recommendations.

Furthermore, Table 1 shows the improvement of our proposed approach compared to the three baseline methods. Our proposed approach TE_PL performs better than the baseline methods both in accuracy and ranking. TE_PL and PL obviously perform better than $\mathrm{CB}$ and $\mathrm{CRS}$ under each metrics. Compared to the PL

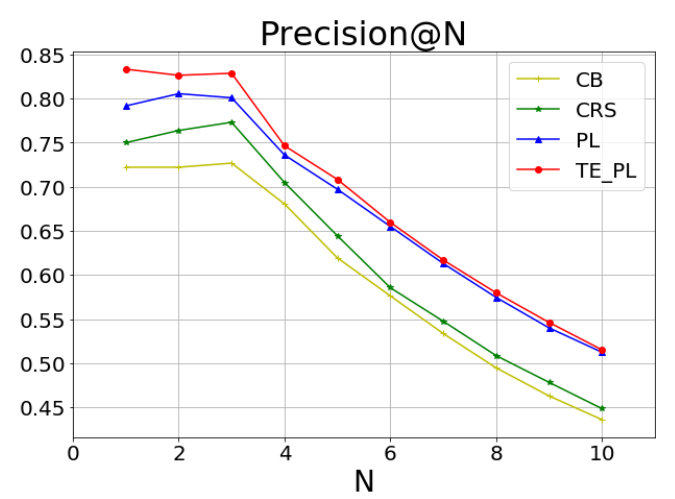

Figure 3. The precision of different methods

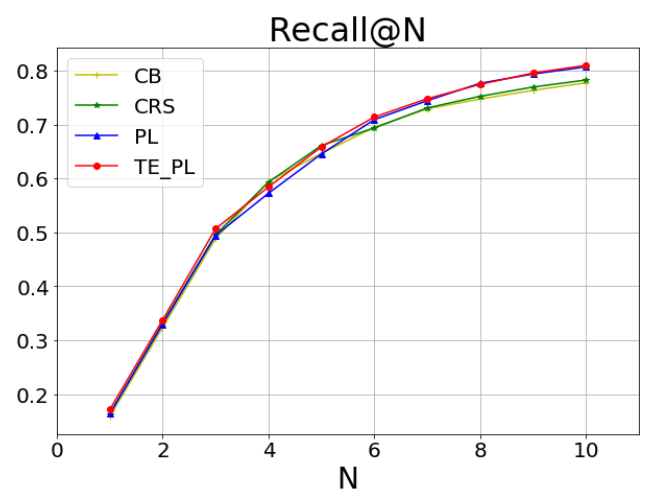

Figure 4. The recall of different methods

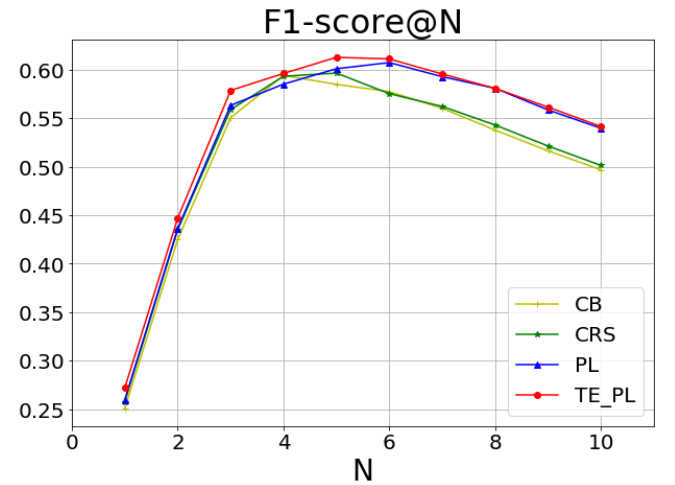

Figure 5. The F1-score of different methods

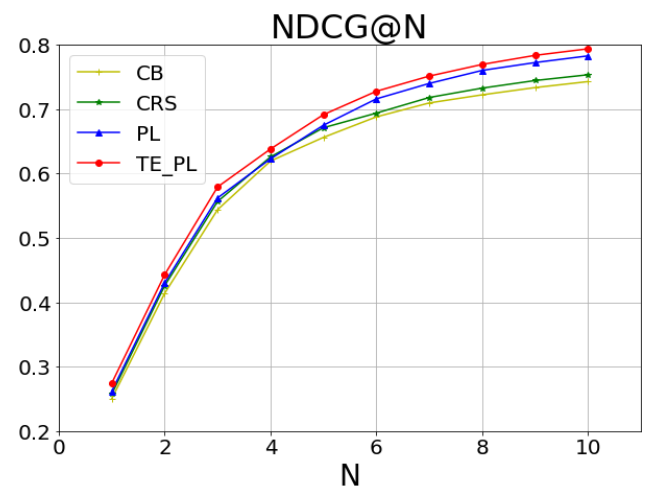

Figure 6. The NDCG of different methods 
method, our method achieves an improvement of $2.29 \%$ in NDCG that reflects ranking performance, while it improves $1.56 \%$ in F1-score. It implicates that the university's and the inventor's trustworthiness are also helpful to improve the recommendation ranking.

Table 1. Average improvement of the proposed approach TE_PL (\%)

\begin{tabular}{|c|c|c|c|c|}
\hline & \multicolumn{4}{|c|}{ TE_PL } \\
\hline $\begin{array}{c}\text { Baseline } \\
\text { methods }\end{array}$ & Precision & Recall & F1-score & NDCG \\
\hline CB & 15.13 & 3.35 & 6.18 & 6.34 \\
\hline CRS & 11.07 & 2.22 & 4.99 & 4.48 \\
\hline PL & 1.83 & 1.50 & 1.56 & 2.29 \\
\hline
\end{tabular}

Table 2. The comparison between PL and TE_PL methods under different trading volume of companies when $\mathrm{K}=3$

\begin{tabular}{|c|c|c|c|c|c|}
\hline Volume & Method & Pre @3 & Rec@3 & F1@3 & $\begin{array}{c}\text { NDCG } \\
\text { @3 }\end{array}$ \\
\hline \multirow{2}{*}{$\begin{array}{c}\text { Small } \\
(10-50)\end{array}$} & PL & 0.794 & 0.612 & 0.677 & 0.666 \\
\cline { 2 - 6 } & TE_PL & $\begin{array}{c}0.818 \\
(3.02)\end{array}$ & $\begin{array}{c}0.628 \\
(2.61)\end{array}$ & $\begin{array}{c}0.696 \\
(2.81)\end{array}$ & $\begin{array}{c}0.686 \\
(3.00)\end{array}$ \\
\hline \multirow{2}{*}{ Large } & PL & 0.824 & 0.113 & 0.194 & 0.228 \\
\cline { 2 - 6 }$(51-500)$ & TE_PL & $\begin{array}{c}0.863 \\
(4.73)\end{array}$ & $\begin{array}{c}0.115 \\
(1.77)\end{array}$ & $\begin{array}{c}0.198 \\
(2.06)\end{array}$ & $\begin{array}{c}0.235 \\
(3.07)\end{array}$ \\
\hline
\end{tabular}

*Note: The numbers in parentheses indicate how much improvement (\%) the proposed method TE_PL has compared to PL method.

Table 3. The comparison between PL and TE_PL methods under different trading volume of companies when $\mathrm{K}=6$

\begin{tabular}{|c|c|c|c|c|c|}
\hline Volume & Method & Pre @6 & Rec@6 & F1@6 & $\begin{array}{c}\text { NDCG } \\
@ 6\end{array}$ \\
\hline \multirow{2}{*}{$\begin{array}{c}\text { Small } \\
(10-50)\end{array}$} & PL & 0.597 & 0.856 & 0.687 & 0.826 \\
\cline { 2 - 6 } & TE_PL & $\begin{array}{c}0.600 \\
(0.50)\end{array}$ & $\begin{array}{c}0.862 \\
(0.70)\end{array}$ & $\begin{array}{c}0.691 \\
(0.58)\end{array}$ & $\begin{array}{c}0.840 \\
(1.69)\end{array}$ \\
\hline \multirow{2}{*}{ Large } & PL & 0.843 & 0.231 & 0.350 & 0.360 \\
\cline { 2 - 6 }$(51-500)$ & TE_PL & $\begin{array}{c}0.853 \\
(1.19)\end{array}$ & $\begin{array}{c}0.232 \\
(0.43)\end{array}$ & $\begin{array}{c}0.352 \\
(0.57)\end{array}$ & $\begin{array}{c}0.365 \\
(1.39)\end{array}$ \\
\hline
\end{tabular}

*Note: The numbers in parentheses indicate how much improvement $(\%)$ the proposed method TE_PL has compared to PL method.

The analysis above shows that our proposed method TE_PL and PL method have similar performance compared to the other two baseline methods. To further explore the appropriate application scenes and the power of TE_PL, we divide the sample companies into two groups according to their patent trading volume. One group of companies with no more than 50 historical assignments is considered small-sized companies. The other group of companies with more than 50 assignments in the past is considered large companies. Then, we evaluate the overall recommendation performance of PL and TE_PL in each group of companies when we recommend three and six academic patents for each company. The results are shown in Table 2 and Table 3. As a whole, TE_PL outperforms PL for both smallsize companies and large-sized companies. Notably, TE_PL achieves greater improvement in recommendation performance when recommending academic patents for small companies in most metrics. Small-sized companies generally lack a professional team to evaluate the potential value of patents, so they tend to depend more on their trust in the corresponding inventor and patent owner before deciding on a transaction $[44,45]$. Thus, the proposed method helps small-sized companies more with academic patent trading.

\section{Conclusion}

This paper proposes a trust-enhanced recommendation approach to encourage academic patent trading. The approach contains a filtering stage and a ranking stage. In the filtering stage, patents whose classification matches the classifications of the target company are extracted as candidates. In the ranking stage, candidate patents are ranked comprehensively based on citation-based connectivity, interpersonal connectivity, and trustworthiness. To evaluate the performance of the proposed recommendation approach, we conduct an offline experiment on representative datasets in the real world. The results demonstrate that our method outperforms the comparison methods, especially in ranking performance. It means that when using our method to make academic patent recommendations, the higher-ranked patents are more likely to attract companies to pay for them. Moreover, we find that the proposed method is more helpful in recommending academic patents to small-sized companies. According to the experiment results, the introduction of trust information to academic patent trading recommendations does improve the recommendation performance.

The contributions and implications of this research are summarized as follows. Firstly, to promote the trading opportunity of academic patents on the online marketplace, we propose a hybrid trustenhanced approach for the academic patent recommendation. The approach extends the general patent recommendation approaches, and considers 
trust relations more comprehensively to make the recommendation results customized for academic patent transactions. In our study, the trust of companies in academic patents is accumulated from their trust in inventors and universities. It is established by the combined action of interpersonal connectivity, and trustworthiness of inventors and universities. Besides, we adopt a hybrid strategy to integrate relevance, connectivity, and trustworthiness before making recommendations. A Personal PageRank model based on tripartite networks is used to measure connectivity based on various relations between companies and patents. Secondly, the proposed approach for academic patent recommendation can be applied to online patent platforms as a service. The analysis of classificationbased similarity and citation-based connectivity enables companies to discover relevant patents among numerous academic patents in the platform. The analysis of interpersonal connectivity and trustworthiness mainly explores the trust relationships between companies and relevant inventors and universities of patents. With the help of the patent recommendation service, companies are expected to be more willing to make a trading negotiation for recommended patents. Thirdly, the recommendation service using the proposed approach is also conducive to disseminating and transferring academic patents. It helps university administrators estimate which patents are more likely to be transferred. According to the approach, administrators in UOTT can adjust their patent transfer strategies and actively participate in the technology transfer activities to increase the likelihood that their university patents will be recommended and transferred.

There are also some limitations and future works. Firstly, our approach considers classification-based relevance, connectivity, and trustworthiness when making academic patent recommendations, but other factors like the company's motivations and industry may also influence academic patent trading. Secondly, more experiments such as an online study will be conducted to furtherly explore the effectiveness of the proposed approach. Thirdly, using our approach, patents that new researchers invent are hard to be recommended and ranked high when there are other patents with similar technological content. New researchers need time to accumulate their trustworthiness and interpersonal connectivity with companies. Similarly, universities that begin to participate in technology transfer activities as new entrants also need transfer experience accumulation. University administrators of new entrants need to spend more effort on finding companies for their patents in the beginning. Finally, this study focuses on the academic patent recommendation. As for the recommendation of other intangible assets, such as academic papers and trademark rights, whether our trust-enhanced recommendation approach can benefit their promotion or trading is also waiting for us to explore further.

\section{References}

[1] A. De Marco, G. Scellato, E. Ughetto, F. Caviggioli, "Global markets for technology: Evidence from patent transactions", Research Policy, 46(9), 2017, 1644-1654.

[2] I.P. McCarthy, K. Ruckman, "Licensing speed: Its determinants and payoffs", Journal of Engineering and Technology Management, 46, 2017, 52-66.

[3] P.M. Swamidass, V. Vulasa, "Why university inventions rarely produce income? Bottlenecks in university technology transfer", The Journal of technology transfer, 34(4), 2009, 343-363.

[4] A. Hagiu, D.B. Yoffie, "The new patent intermediaries: platforms, defensive aggregators, and superaggregators", Journal of Economic Perspectives, 27(1), 2013, 45-66.

[5] T. Yanagisawa, D. Guellec, "The emerging patent marketplace", 2009.

[6] Q. Wang, W. Du, J. Ma, X. Liao, "Recommendation Mechanism for Patent Trading Empowered by Heterogeneous Information Networks", International Journal of Electronic Commerce, 23(2), 2019, 147-178.

[7] J. Bih-Huang, C.-Y. Chu, "The Value Creation Model of Patent Market Intermediaries", Global Journal of Business Research, 9(2), 2015, 75-86.

[8] I. Troy, R. Werle, "Uncertainty and the Market for Patents", 2008.

[9] M. Kani, K. Motohashi, "Understanding the technology market for patents: New insights from a licensing survey of Japanese firms", Research Policy, 41(1), 2012, 226-235.

[10] R. Lowe, "Entrepreneurship and information asymmetry: theory and evidence from the University of California", Unpublished working paper, Haas School of Business, 2001.

[11] A.J. Trappey, C.V. Trappey, C.-Y. Wu, C.Y. Fan, Y.-L. Lin, "Intelligent patent recommendation system for innovative design collaboration", Journal of Network and Computer Applications, 36(6), 2013, 1441-1450.

[12] S. Bashir, A. Rauber, "Improving retrievability of patents in prior-art search", in: European Conference on Information Retrieval, Springer, 2010, pp. 457-470.

[13] F. Wang, L. Lin, "Query construction based on concept importance for effective patent retrieval", in: 2015 12th International Conference on Fuzzy Systems and Knowledge Discovery (FSKD), IEEE, 2015, pp. 14551459.

[14] P. Mahdabi, F. Crestani, "The effect of citation analysis on query expansion for patent retrieval", Information retrieval, 17(5-6), 2014, 412-429.

[15] S. Oh, Z. Lei, W.-C. Lee, P. Mitra, J. Yen, “CV-PCR: a context-guided value-driven framework for patent citation recommendation", in: Proceedings of the 22nd 
ACM international conference on Information \& Knowledge Management, ACM, 2013, pp. 2291-2296.

[16] T.-y. Fu, Z. Lei, W.-C. Lee, "Patent citation recommendation for examiners", in: 2015 IEEE International Conference on Data Mining, IEEE, 2015, pp. 751-756.

[17] X. Jin, S. Spangler, Y. Chen, K. Cai, R. Ma, L. Zhang, $\mathrm{X}$. $\mathrm{Wu}, \mathrm{J}$. Han, "Patent maintenance recommendation with patent information network model", in: 2011 IEEE 11th International Conference on Data Mining, IEEE, 2011, pp. 280-289.

[18] X.-j. He, Y. Dong, Z. Zhen, Y.-y. Wu, G.-r. Jiang, X. Meng, S. Ma, "Weighted meta paths and networking embedding for patent technology trade recommendations among subjects", Knowledge-Based Systems, 184, 2019, 104899.

[19] B. Six, E. Zimmeren van, F. Popa, C. Frison, "Trust and social capital in the design and evolution of institutions for collective action", International Journal of the Commons, 9(1), 2015, 151-176.

[20] J. Lu, D. Wu, M. Mao, W. Wang, G. Zhang, "Recommender system application developments: a survey", Decision Support Systems, 74, 2015, 12-32.

[21] J. Chen, J. Chen, S. Zhao, Y. Zhang, J. Tang, "Exploiting word embedding for heterogeneous topic model towards patent recommendation", Scientometrics, 125(3), 2020, 2091-2108.

[22] W. Shalaby, W. Zadrozny, "Patent retrieval: a literature review", Knowledge and Information Systems, 2019, 130.

[23] Y.-L. Chen, Y.-T. Chiu, “An IPC-based vector space model for patent retrieval", Information Processing \& Management, 47(3), 2011, 309-322.

[24] F. Wang, L. Lin, "Exploiting semantic knowledge base for patent retrieval", in: 2017 13th International conference on natural computation, fuzzy systems and knowledge discovery (ICNC-FSKD), IEEE, 2017, pp. 2195-2200.

[25] L. Helmers, F. Horn, F. Biegler, T. Oppermann, K.-R. Müller, "Automating the search for a patent's prior art with a full text similarity search", PloS one, 14(3), 2019, $\mathrm{e} 0212103$.

[26] S. Ma, C. Zhang, X. Liu, "A review of citation recommendation: from textual content to enriched context", Scientometrics, 122(3), 2020, 1445-1472.

[27] K. Ruckman, I. McCarthy, "Why do some patents get licensed while others do not?", Industrial and Corporate Change, 26(4), 2017, 667-688.

[28] Q. Shambour, J. Lu, "A trust-semantic fusion-based recommendation approach for e-business applications", Decision Support Systems, 54(1), 2012, 768-780.

[29] Q. Shambour, J. Lu, “A hybrid trust - enhanced collaborative filtering recommendation approach for personalized government - to - business e - services”, International Journal of Intelligent Systems, 26(9), 2011, 814-843.

[30] M. Jamali, M. Ester, "Trustwalker: a random walk model for combining trust-based and item-based recommendation", in: Proceedings of the 15th ACM SIGKDD international conference on Knowledge discovery and data mining, ACM, 2009, pp. 397-406.
[31] J. Jung, W. Jin, L. Sael, U. Kang, "Personalized ranking in signed networks using signed random walk with restart", in: 2016 IEEE 16th International Conference on Data Mining (ICDM), IEEE, 2016, pp. 973-978.

[32] W. Yuan, L. Shu, H.-C. Chao, D. Guan, Y.-K. Lee, S. Lee, "ITARS: trust-aware recommender system using implicit trust networks", IET communications, 4(14), 2010, 1709-1721.

[33] F. Caviggioli, E. Ughetto, "The drivers of patent transactions: corporate views on the market for patents", R\&d Management, 43(4), 2013, 318-332.

[34] P. Bedi, R. Sharma, "Trust based recommender system using ant colony for trust computation", Expert Systems with Applications, 39(1), 2012, 1183-1190.

[35] T. Yu, J. Guo, W. Li, H.J. Wang, L. Fan, "Recommendation with diversity: an adaptive trustaware model", Decision Support Systems, 2019, 113073.

[36] Y.-M. Li, C.-T. Wu, C.-Y. Lai, “A social recommender mechanism for e-commerce: Combining similarity, trust, and relationship", Decision Support Systems, 55(3), 2013, 740-752.

[37] M. Perkmann, V. Tartari, M. McKelvey, E. Autio, A. Broström, P. D'Este, R. Fini, A. Geuna, R. Grimaldi, A. Hughes, "Academic engagement and commercialisation: A review of the literature on university-industry relations", Research policy, 42(2), 2013, 423-442.

[38] E. Albats, I. Fiegenbaum, J.A. Cunningham, "A micro level study of university industry collaborative lifecycle key performance indicators", The Journal of Technology Transfer, 43(2), 2018, 389-431.

[39] F. Rossi, A. Rosli, "Indicators of university-industry knowledge transfer performance and their implications for universities: evidence from the United Kingdom", Studies in Higher Education, 40(10), 2015, 1970-1991.

[40] M. Hemmert, L. Bstieler, H. Okamuro, "Bridging the cultural divide: Trust formation in university-industry research collaborations in the US, Japan, and South Korea", Technovation, 34(10), 2014, 605-616.

[41] E. Giaretta, "The trust "builders" in the technology transfer relationships: an Italian science park experience", The Journal of Technology Transfer, 39(5), 2014, 675-687.

[42] M. Perkmann, A. Neely, K. Walsh, "How should firms evaluate success in university-industry alliances? A performance measurement system", R\&D Management, 41(2), 2011, 202-216

[43] M. Usman, G. Mustafa, M.T. Afzal, "Ranking of author assessment parameters using Logistic Regression", Scientometrics, 2020, 1-19.

[44] K. Lee, D. Go, I. Park, B. Yoon, "Exploring suitable technology for small and medium-sized enterprises (SMEs) based on a hidden markov model using patent information and value chain analysis", Sustainability, 9(7), 2017, 1100.

[45] K. Blomqvist, P. Hurmelinna, R. Seppänen, "Playing the collaboration game right - balancing trust and contracting", Technovation, 25(5), 2005, 497-504. 